\title{
NOMINATA DE PARECERISTAS 2017
}

\section{a) Pareceristas "ad hoc" (externos)}

Adriano Lima: Faculdade Cristã de Curitiba, FCC adriano.lima.66@hotmail.com

Adriano Holanda: Universidade Federal do Paraná, UFPR aholanda@yahoo.com

Afonso Tadeu Murad: Faculdade Jesuíta de Filosofia e Teologia, FAJE amurad@marista.edu.br

Agemir de Carvalho Dias: Faculdade Evangélica do Paraná, FEPAR agemir-dias@uol.com.br

Andreia Serrato: Escola de Educação e Humanidades, EEHU da PUCPR andreia.serrato@uol.com.br

Antonio Manzatto: Pontifícia Universidade Católica de São Paulo, PUC-SP pastbrasilandia@gmail.com

Antônio Renato Gusso: Faculdades "Batista" do Paraná, FABAPAR renatogusso@hotmail.com

Agemir de Carvalho Dias: Faculdade Evangélica do Paraná, FEPAR agemir@terra.com.br

Boris Neff Ulloa: Pontifícia Universidade Católica de São Paulo, PUC-SP banefulloa@gmail.com

Cirlene Cristina de Sousa: Universidade Estadual de Minas Gerais, UEMG cirlenesousa@yahoo.com.br

Claudete Ulrich: Faculdade Unidade de Vitória, UNIDA claudete@faculdadeunida.com.br

Cleusa Caldeira: Faculdade Jesuíta de Filosofia e Teologia, FAJE cleucaldeira@gmail.com 
David Mesquiati de Oliveira: Faculdade Unidade de Vitória, UNIDA

david@faculdadeunida.com.br

David Vides: Universidad La Salle, UNILASALLE (Colombia)

demmanuel01@gmail.com

Edinei da Rosa Cândido: Faculdade Católica de Santa Catarina, FACASC

edinei@tiscalinet.it

Emerli Schlogl: Universidade Estadual do Paraná, UNESPAR

emerli.schlogl@gmail.com

Euler Renato Westphal: Faculdade Luterana de Teologia, FLT

eulerrw@brturbo.com.br

Fabiano Incerti: Instituto Ciência e Fé PUC PR, CIÊNCIA \& FÉ incerti.fabiano@pucpr.br.

Fátima Regina Machado: Pontifícia Universidade Católica de São Paulo, PUC-SP fatimaregina@usp.br

Geraldo José de Paiva: Universidade de São Paulo, USP

gjdpaiva@usp.br

Gerson Joni Fischer: Faculdades "Batista" do Paraná, FABAPAR

gersonjf@hotmail.com

Gleyds Silva Domingues: Faculdades "Batista” do Paraná, FABAPAR

gleyds@ftbp.com.br

Jaci Candiotto: Programa de Pós-Graduação em Direitos Humanos, PPGDH da PUCPR j.candiotto@pucpr.br

James Farris: Universidade Metodista de São Paulo, UMESP

theology@uol.com.br

José Adalberto Vanzella: Faculdade Dehoniana, DEHONIANA

adalbertovanzella@gmail.com 
José Francisco M. H. Bairrão: Universidade de São Paulo, USP

jfbairrao@ffclrp.usp.br

José Neivaldo de Sousa: Faculdades "Batista" do Paraná, FABAPAR

neivaldojs@gmail.com

Josemar Maciel: Universidade Católica Dom Bosco de Campo Grande, UCDB

maciel50334@yahoo.com.br

Laude Brandenburg: Faculdades EST, EST

laude@est.edu.br

Letícia Oliveira Alminhana: Pontifícia Universidade Católica do Rio Grande do Sul, PUCRS leticia.alminhana@pucrs.br

Luciana Fernandes Marques: Universidade Federal do Rio Grande do Sul, UFRGS

luciana.marques@ufrgs.br

Marcelo Batalioto: Faculdade Dehoniana, DEHONIANA

batalioto@uol.com.br

Marcos Aurélio Fernandes: Universidade de Brasília, UnB

framarcosaurelio@hotmail.com

Maria Aparecida de Almeida: Universidade Metodista de São Paulo, UMESP

mcidalmeida@hotmail.com

Maria Freire da Silva: Pontifícia Universidade Católica de São Paulo, PUC-SP

mfreire.silva@pucsp.br

Maria Teresa de F. Cardoso: Pontifícia Universidade Católica do Rio de Janeiro, PUC-Rio mtfcardoso@puc-rio.br

Mário Marcelo Coelho: Faculdade Dehoniana, DEHONIANA

marioscj@uol.com.br

Marta Helena de Freitas: Universidade Católica de Brasília, UCB

mhelena@ucb.br

Matthias Grenzer: Pontifícia Universidade Católica de São Paulo, PUC-SP

mgrenzer@pucsp.br 
Miguel Farias: Coventry University, COVENTRY (United Kingdom)

miguel.farias@wolfson.ox.ac.uk

Patricio A. Merino Beas: Centro de Estudios Bíblicos, Teológicos y Pastorales de América Latina, CEBITEPAL (Colombia) cebitepal.teologia@celam.org

Pedro Iwashita: Pontifícia Universidade Católica de São Paulo, PUC-SP iwashita2001@gmail.com

Ricardo Quadros Gouvea: Universidade Presbiteriana Mackenzie, MACKENZIE ricardoqgouvea@gmail.com

Roberto E. Zwetsch: Faculdades EST, EST

rezwetsch@gmail.com

Rodrigo Polanco: Pontificia Universidad Católica de Chile, PUC Chile (Chile) rpolanco@uc.cl

Ronaldo Sathler-Rosa: Society for Intercultural Pastoral Care and Counseling, SIPCC (Deutschland) ronaldo.sathler@gmail.com

Rui Luís Rodrigues: Universidade Estadual de Campinas, UNICAMP ruiluis@unicamp.br

Tarcísio Justino Loro: Pontifícia Universidade Católica de São Paulo, PUC-SP tjloro@pucsp.br

Thiago Antonio Avellar de Aquino: Universidade Federal da Paraíba, UFPB logosvitae@ig.com.br

Valério Guilherme Schaper: Faculdades EST, EST

valerio@est.edu.br

Vítor Galdino Feller: Faculdade Católica de Santa Catarina, FACASC

vitorfeller@arquifln.org.br

Wagner Ferreira da Silva: Faculdade Dehoniana, DEHONIANA

wagnerfcn@hotmail.com 
Wellington Zangari: Universidade de São Paulo, USP

w.z@usp.br

\section{b) Pareceristas docentes do PPGT (internos)}

Agenor Brighenti: Pontifícia Universidade Católica do Paraná, PUCPR agenor.brighenti@pucpr.br

Clélia Peretti: Pontifícia Universidade Católica do Paraná, PUCPR cpkperetti@gmail.com

Elias Wolff: Pontifícia Universidade Católica do Paraná, PUCPR elias.wolff@facasc.edu.br

Luiz Alexandre Solano Rossi: Pontifícia Universidade Católica do Paraná, PUCPR luizalexandrerossi@yahoo.com.br

Luiz José Dietrich: Pontifícia Universidade Católica do Paraná, PUCPR luizdietrich@ig.com.br

Marcio Fernandes: Pontifícia Universidade Católica do Paraná, PUCPR marciovisconde@yahoo.com.br

Mário Sanches: Pontifícia Universidade Católica do Paraná, PUCPR m.sanches@pucpr.brR

Vicente Artuso: Pontifícia Universidade Católica do Paraná, PUCPR vicenteartuso@gmail.com

Waldir Souza: Pontifícia Universidade Católica do Paraná, PUCPR wacasouza@yahoo.com.br 